\title{
NOUVELle
}

\section{Les transporteurs de cations organiques, nouvelles cibles thérapeutiques contre la dépression}

\author{
Sophie Gautron ${ }^{1}$, Nicolas Pietranscosta ${ }^{2}$
}

\author{
${ }^{1}$ Neuroscience Paris-Seine, Institut de biologie Paris-Seine, \\ Sorbonne Université, CNRS UMR8246, Inserm U1130, \\ 9 quai Saint Bernard, 75252 Paris, France. \\ ${ }^{2}$ Laboratoire des Biomolécules, Sorbonne Université, \\ CNRS UMR7203, Paris, France. \\ sophie.gautron@upmc.fr
}

$>$ Les troubles dépressifs touchent 10 à $20 \%$ de la population mondiale [1]. Cette grave instabilité de l'humeur affecte également la cognition, la motivation et le comportement, et pèse lourdement sur la qualité de vie des individus. Les troubles dépressifs sont associés à une surmortalité, un risque de suicide élevé et diverses comorbidités, et ont un impact social et économique considérable. La dépression majeure est une maladie complexe sur le plan étiologique, impliquant de multiples facteurs de risque génétiques et environnementaux. L'arsenal thérapeutique disponible pour lutter contre cette maladie englobe la pharmacothérapie, les psychothérapies comportementales et cognitives, et la stimulation magnétique transcranienne ou la stimulation cérébrale profonde, plus rarement utilisées. À ce jour, les antidépresseurs restent le traitement de première intention contre la dépression majeure, mais leur efficacité varie considérablement d'un individu à l'autre.

Pendant plus d'un demi-siècle, les troubles dépressifs ont été traités principalement à l'aide d'une pharmacopée ciblant la neurotransmission aminergique. Ce constat a donné naissance à I'hypothèse monoaminergique, qui stipule l'existence, chez les sujets atteints, d'un déséquilibre de la transmission sérotoninergique et noradrénergique, que l'on pourrait corriger avec des médicaments modulant ces voies de neurotransmission [2]. De fait, la plupart des antidépresseurs prescrits actuellement sont des inhibiteurs de la recapture de la sérotonine (5-hydroxytryptamine, $5-\mathrm{HT}$ ) et, pour certains, de la norépinéphrine (NE), ciblant des transporteurs de haute affinité pour ces monoamines situés dans les terminaisons aminergiques. Ces inhibiteurs de recapture présentent toutefois un long délai d'action, et environ un tiers des patients ne répond pas de manière satisfaisante après deux tentatives de traitement avec différentes catégories d'antidépresseurs [3]. L'identification de nouvelles pistes thérapeutiques constitue donc un défi majeur pour la recherche en santé mentale.

Dans ce contexte, notre équipe s'est intéressée aux transporteurs de cations organiques (organic cation transporters, OCT), des transporteurs atypiques des monoamines. Les OCT sont des transporteurs polyspécifiques de type facilitateur, qui participent à l'absorption et à la clairance de divers composés physiologiques et de xénobiotiques dans le système nerveux et les organes périphériques. Ces transporteurs ont une faible affinité (de l'ordre du mM) pour les amines biogènes, $5-H T, N \varepsilon$, dopamine et histamine [4]. Deux transporteurs de cette famille, 0СT2 et OCT3, sont exprimés dans le système nerveux central, où ils interviennent dans l'anxiété, la réponse au stress et l'action des antidépresseurs [4-7]. Ces OCT constitueraient un système alternatif de clairance des monoamines, dans des sites pauvres en transporteurs de haute affinité à distance des varicosités aminergiques, ou encore en cas de saturation de ces derniers, lors de traitements avec des antidépresseurs (Figure I).
En accord avec cette fonction, ces OCT présentent une distribution très étendue dans le cerveau (contrairement aux transporteurs de haute affinité), et sont retrouvés dans une grande variété de types neuronaux et parfois dans des astrocytes, dans la quasi-totalité des régions recevant des projections aminergiques [4]. Nous avons tiré parti des expertises combinées de deux équipes de recherche, en neurosciences et en chimie, pour développer une prodrogue ciblant les $\mathrm{OCT}$, en faisant I'hypothèse qu'elle pourrait exercer un effet antidépresseur par des mécanismes différents du blocage des transporteurs de haute affinité.

Les inhibiteurs connus des OCT couvrent une large gamme de composés, d'efficacité variée et souvent de faible sélectivité, comme des analogues de nucléosides, des dérivés cyanine et des hormones [4]. Nous avons choisi le disprocynium 24 (D24), un dérivé d'isocyanine, comme chef de file pour des expériences de pharmaco-modulation [8] (Figure 2). Le D24 est un inhibiteur assez sélectif des OCT, qui interagit avec un site de liaison de haute affinité de ces transporteurs, avec des constantes d'affinité comprises entre 15 et 280 nM pour 0CT2 et 0CT3. Néanmoins, il existe des obstacles importants à l'utilisation préclinique et clinique de D24, puisqu'il inhibe également les récepteurs adrénergiques $\alpha 1$ et $\alpha 2$ [9] et bloque la clairance des catécholamines dans les tissus périphériques. De plus, les dérivés cyanine traversent mal la barrière hématoencéphalique. Nous avons résolu ces 
Antidépresseurs conventionnels

Ciblent les transporteurs de recapture à haute affinité, localisés sur les neurones et terminaisons aminergiques

\section{Cyanome}

Cible les $\mathrm{OCT}$, polyspécifiques et exprimés dans les régions recevant les projections aminergiques

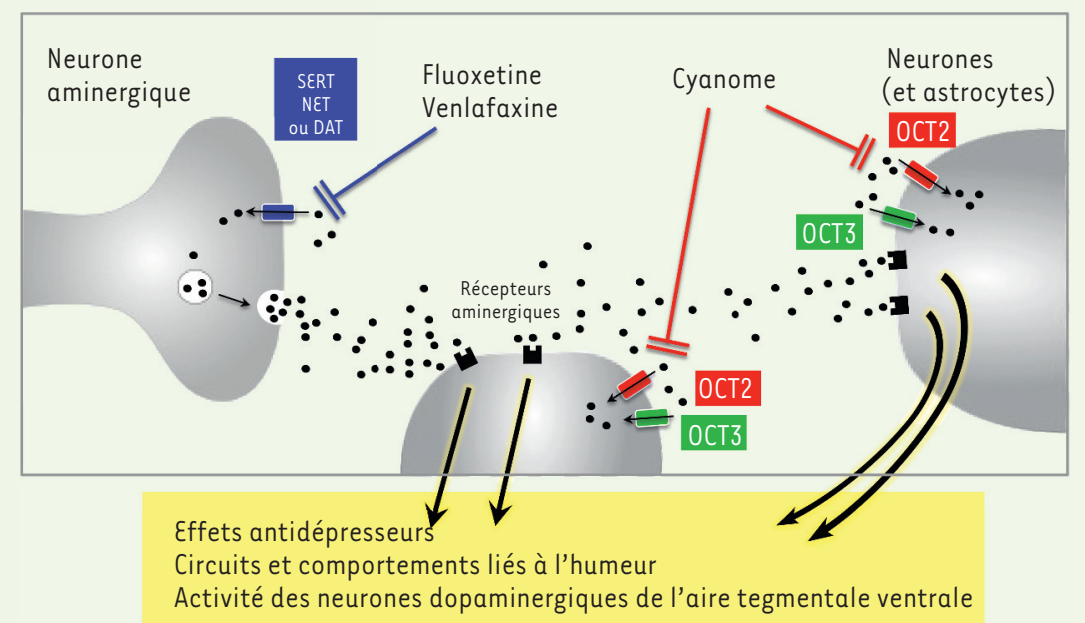

Figure 1. Rôle des transporteurs de cations organiques (OCT) dans la clairance des monoamines dans le cerveau et mécanisme d'action du cyanome. Les monoamines (sérotonine, norépinéphrine et dopamine) agissent sur des récepteurs spécifiques. Elles sont libérées par les varicosités aminergiques, et sont réinternalisées dans ces terminaisons par des transporteurs de haute affinité (SERT, serotonin transporter; NET, norepinephrine transporter; DAT, dopamine transporter). L'inhibition pharmacologique de ces transporteurs par les médicaments antidépresseurs conventionnels, tels que la fluoxétine ou la venlafaxine, augmente la concentration extracellulaire des monoamines. Les transporteurs OCT2 et OCT3, exprimés par de nombreuses sous-populations neuronales (et certains astrocytes) dans les régions recevant des projections aminergiques, contribuent également à la clairance des monoamines. L'inhibition des OCT par le cyanome provoque une diminution de cette clairance, augmentant les concentrations extracellulaires en monoamines dans ces régions, et induisant à long terme des effets antidépresseurs robustes.

problèmes en combinant une modélisation moléculaire et une approche par prodrogue, et avons ainsi obtenu un composé avec une sélectivité accrue pour 0CT2, capable de pénétrer dans le cerveau [8].

Nous avons généré un modèle tridimensionnel des récepteurs $\alpha$-adrénergiques à partir de données cristallographiques obtenues pour le récepteur $\beta$-adrénergique, et modifié les groupements de D24 qui interagissaient avec le récepteur adrénergique $\alpha 2 C$, afin de diminuer les interactions avec ce dernier (Figure 2). In silico (dans un modèle dérivé des données cristallographiques de la lactose perméase Lac y), cette cyanine modifiée (appelée cyanome) conserve ses interactions avec 0CT2 [8]. La pertinence des interactions ainsi modélisées a été vérifiée par des expériences de transport et de liaison in vitro, évaluant l'affinité du cyanome pour OCT2 et pour les récepteurs $\alpha$-adrénergiques. Le cyanome a ensuite été modifié en une prodrogue, le H2-cyanome (Figure 2), pour permettre sa diffusion passive dans le parenchyme cérébral et son activation selon un mécanisme d'oxydo-réduction. L'analyse par spectrométrie de masse du sang et du cerveau chez la souris confirme que le $\mathrm{H} 2$-cyanome diffuse dans le cerveau [8].
Pour évaluer le potentiel antidépresseur du H2-cyanome, nous avons utilisé un modèle validé de dépression chronique, en le comparant à un antidépresseur conventionnel, la fluoxétine (Prozac ${ }^{\circledR}$ ). Chez les rongeurs, l'exposition prolongée à la corticostérone induit un ensemble d'anomalies qui miment différents symptômes de la dépression dans l'espèce humaine, comme l'anhédonie, l'anxiété, l'aversion sociale et les troubles de la mémoire. Les anomalies comportementales persistantes induites par la corticostérone dans ce modèle animal peuvent être améliorées par un traitement prolongé - mais non par un traitement de courte durée - avec des antidépresseurs conventionnels, une particularité qui renforce la pertinence de ces modèles. Dans le modèle murin, l'administration prolongée du H2-cyanome a un effet favorable sur l'ensemble de ces anomalies caractéristiques (Figure 1). Notamment, le H2-cyanome agit plus rapidement (en 10 jours) sur l'anhédonie que la fluoxétine, et a un effet anxiolytique plus prononcé [8].

Nous supposons que les interactions réduites du $\mathrm{H} 2$-cyanome avec les récepteurs $\alpha$-adrénergiques et sa pénétration efficace dans le cerveau sont responsables de ses effets antidépresseurs robustes. Les récepteurs $\alpha$-adrénergiques sont impliqués dans le contrôle de la pression artérielle, de la fréquence et de la contractilité cardiaques, et limiter leur inhibition peut donc être bénéfique pour éviter des effets cardio-vasculaires indésirables. Il est important de noter que l'interaction du $\mathrm{H} 2$-cyanome avec les récepteurs $\alpha$-adrénergiques centraux pourrait également contribuer à son efficacité anti-dépressive, mais il est difficile de prédire dans quelle mesure l'inhibition de certains soustypes de ces récepteurs constitue un avantage. Compte tenu de la diversité des fonctions attribuées aux récepteurs $\alpha$-adrénergiques, à la fois dans les tissus périphériques et dans le cerveau, l'optimisation du H2-cyanome nécessi- 


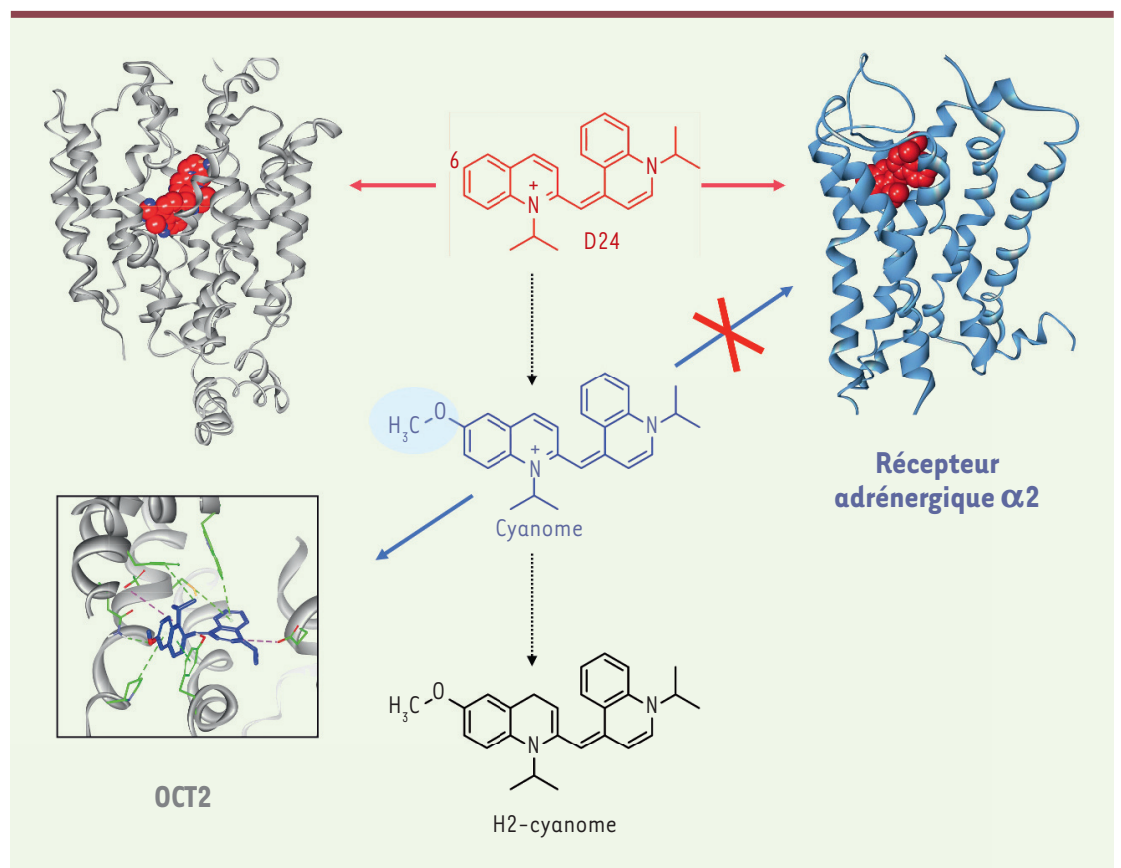

Figure 2. Conception d'un inhibiteur des transporteurs de cations organiques. Les positions d'interaction du disprocynium 24 (D24) avec le transporteur de cations organiques 2 (OCT2) et le récepteur adrénergique $\alpha 2$ ont été définies par modélisation 3D du complexe ligand/ transporteur. Sur la base de ces interactions, un nouveau composé, le cyanome, a été conçu et synthétisé afin d'améliorer la sélectivité pour OCT2 (vue rapprochée). Le cyanome a ensuite été converti en $\mathrm{H} 2$-cyanome, une prodrogue qui peut diffuser dans le cerveau pour y être transformée en cyanome.

tera d'affiner sa sélectivité pour les OCT et certains de ces récepteurs.

Un effet du H2-cyanome sur l'activité de l'aire tegmentale ventrale du cerveau peut être à l'origine de son effet rapide sur l'anhédonie. L'administration prolongée du $\mathrm{H} 2$-cyanome induit en effet une augmentation de la fréquence de décharge des neurones dopaminergiques de cette région. L'activation de ces neurones a été associée aux processus de récompense et à la motivation [10], souvent altérés au cours d'une dépression. Notre étude a révélé également l'existence d'effets robustes du H2-cyanome sur des voies de signalisation intracellulaires impliquées dans le contrôle de l'humeur et la réponse aux médicaments antidépresseurs, en particulier sur la MAP kinase ERK (extracellular signal-regulated kinase) $1 / 2$, GSK3 $\beta$ (glycogen synthase kinase- $3 \beta$ ) et la rante depuis plusieurs années. Nos résultats précliniques fournissent la «preuve de concept» du potentiel antidépresseur d'un ligand des OCT, mais des expériences complémentaires seront nécessaires pour affiner ses propriétés et déterminer comment il peut pallier les faiblesses des traitements actuels (délai d'action, efficacité limitée et effets indésirables). Ces travaux pourront fournir un cadre conceptuel et mécanistique pour le développement de nouvelles classes de médicaments pour traiter la dépression et d'autres troubles psychiatriques, tels que l'anxiété. $\diamond$ Organic cation transporters as novel therapeutic targets for depression

\section{LIENS D'INTÉRÊT}

Sophie Gautron et Nicolas Pietrancosta sont coinventeurs d'un brevet européen portant sur les dérivés du H2-cyanome.

\section{RéFÉRENCES}

1. Disease GBD, Injury I, Prevalence C. Global, regional, and national incidence, prevalence, and years lived with disability for 328 diseases and injuries for 195 countries, 1990-2016: a systematic analysis for the global burden of disease study 2016. Lancet 2017 . 390 : 1211-59.

2. Wong ML, Licinio J. Research and treatment approaches to depression. Nat Rev Neurosci 2001 ; 2 343-51.

3. Rush AJ, Trivedi MH, Wisniewski SR, et al. Acute and longer-term outcomes in depressed outpatients requiring one or several treatment steps: a STAR ${ }^{D}$ report. Am J Psychiatry 2006 ; 163 : 1905-17.

4. Courousse T, Gautron S. Role of organic cation transporters (OCTs) in the brain. Pharmacol Ther 2015 ; 146C : 94-103.

5. Amphoux A, Vialou V, Drescher $\varepsilon$, et al. Differential pharmacological in vitro properties of organic cation transporters and regional distribution in rat brain. Neuropharmacology $2006 ; 50: 941-52$

6. Bacq A, Balasse L, Biala G, et al. Organic cation transporter 2 controls brain norepinephrine and serotonin clearance and antidepressant response. Mol Psychiatry 2012; 17 : 926-39.

7. Courousse T, Bacq A, Belzung C, et al. Brain organic cation transporter 2 controls response and vulnerability to stress and GSK3 $\beta$ signaling. Mol Psychiatry $2015 ; 20: 889-900$.

8. Orrico-Sanchez A, Chausset-Boissarie L, Alves de Sousa $R$, et al. Antidepressant efficacy of a selective organic cation transporter blocker in a mouse model of depression. Mol Psychiatry $2020 ; 25$ : 1245-59.

9. Amphoux A, Millan MJ, Cordi A, et al. Inhibitory and facilitory actions of isocyanine derivatives at human and rat organic cation transporters 1, 2 and 3: a comparison to human $\alpha 1$ - and $\alpha 2$-adrenoceptor subtypes. Eur J Pharmacol $2010 ; 634$ : 1-9.

10. Morales M, Margolis $\varepsilon B$. Ventral tegmental area: cellular heterogeneity, connectivity and behaviour. Nat Rev Neurosci 2017 ; 18 : 73-85 